\title{
Ridge Localizations and Networks in Thin Films Compressed by the Incremental Release of a Large Equi-biaxial Pre-stretch in the Substrate
}

\section{Citation}

Takei, Atsushi, Lihua Jin, John W. Hutchinson, and Hiroyuki Fujita. 2014. "Ridge Localizations and Networks in Thin Films Compressed by the Incremental Release of a Large Equi-Biaxial Pre-Stretch in the Substrate." Advanced Materials 26 (24) (March 31): 4061-4067. doi:10.1002/ adma.201306162.

\section{Published Version}

doi:10.1002/adma.201306162

\section{Permanent link}

http://nrs.harvard.edu/urn-3:HUL.InstRepos:14023478

\section{Terms of Use}

This article was downloaded from Harvard University's DASH repository, and is made available under the terms and conditions applicable to Open Access Policy Articles, as set forth at http:// nrs.harvard.edu/urn-3:HUL.InstRepos:dash.current.terms-of-use\#OAP

\section{Share Your Story}

The Harvard community has made this article openly available.

Please share how this access benefits you. Submit a story.

Accessibility 


\section{Ridge localizations and networks in thin films compressed by incremental release of large equi-biaxial substrate pre-stretch}

By Atsushi Takei ${ }^{1}$, Lihua Jin ${ }^{2}$, John W. Hutchinson ${ }^{2}$, and Hiroyuki Fujita ${ }^{1}$.

Dr. A. Takei, Prof. H. Fujita

${ }^{1}$ Institute of Industrial Science, The University of Tokyo, 4-6-1 Komaba, Meguro-ku, Tokyo 153-8505 (Japan)

E-mail: atakei,fujita@iis.u-tokyo.ac.jp

L. Jin, Prof. J. W. Hutchinson

${ }^{2}$ School of Engineering and Applied Sciences, Kavli Institute, Harvard University, 29 Oxford Street, Cambridge, MA 02138 (USA).

Keywords: Thin film buckling, wrinkling, ridge localization, substrate pre-stretch, ridge networks

The intriguing buckling patterns exhibited by bi-layer systems composed of a thin stiff film bonded to a thick elastomeric substrate have been studied extensively in recent years both experimentally and theoretically. Under compression, the film is constrained by the substrate and buckles with a wavelength that is usually large compared to the film thickness yet small compared to substrate thickness. The most common patterns observed are the sinusoidal wrinkle mode when compression in one direction is dominant ${ }^{[1]}$, and the herringbone mode ${ }^{[2,3]}$ and the labyrinth mode ${ }^{[2-4]}$ under states of equi-biaxial compression. The present paper is part of the ongoing research effort in the soft materials community to produce and manipulate specific buckling patterns ${ }^{[5,6]}$. Most of the previous work has focused on buckling patters of bi-layer systems under small compression. Recent work, however, has demonstrated that large compression of a bi-layer system can engender other surface patterns such as period-doubling ${ }^{[7]}$ and folding ${ }^{[8,9]}$, opening the way for the creation of a wider array of surface patterns. These post-wrinkling modes involve the large strain behavior of the substrate and geometrical nonlinearity of the films in an essential way.

The focus of the present paper is on the most recent of the post-wrinkling modes to be discovered, the ridge localization mode. While folds and creases protrude into the substrate, the ridge is a large amplitude localization that pushes out from the substrate. This mode 
should be characterized as a localization because the large amplitude of the ridge is fed by flattening the nearby wrinkle undulations resulting in ridges that are separated from each other by expanses of relatively flat film. Thus, a distinct feature of the transition from wrinkles to ridges is the abrupt increase of spacing from crest to crest. The ridge localization was predicted to occur $^{[10]}$, and has been observed experimentally ${ }^{[5,6,11]}$, only when a relatively large pre-stretch is imposed on the elastomeric substrate prior to film attachment. Controlled incremental release of the pre-stretch of the thick substrate then imposes compression on the film which drives buckling. The large substrate pre-stretch favors the formation of the ridge mode ${ }^{[10]}$. The buckling pattern reported in this paper consists of localized ridge lines separated by relatively flat patches of film. Exploitation of high pre-stretch produces these new high aspect convex structures which have potential for expanding the range of surface morphologies for applications such as smart adhesion ${ }^{[12]}$, hydrophobic surfaces ${ }^{[13-15]}$, and templates for micro-patterning ${ }^{[16]}$. The present paper features a method for applying equibiaxial pre-stretch and release and thereby complements recent related work on ridge formation which has primarily been limited to uni-axial or plane strain pre-stretch ${ }^{[5,10,11,17]}$. The present paper also highlights the underlying characteristics and nature of ridges including emergence and evolution aspects still unclear due to the highly non-linear behavior of the system caused by high pre-stretch. For the system and approach employed here delamination $^{[5,18,19]}$ and cracking ${ }^{[20]}$ of the thin film, which often accompany wrinkling instabilities, have been avoided.

Thus, in this paper, the transition to the ridge buckling morphology is studied in a bi-layer system comprised of a thin stiff film attached to a relatively thick compliant substrate. The substrate is subject to a large equi-biaxial pre-stretch prior to film attachment and then the bilayer is incrementally unloaded producing equi-biaxial compression in the film. The experiments are carried out using a specially designed micro-fluidic loading system. The system enables equi-biaxial pre-stretch and incremental compression simply by changing 
pressure inside the micro-fluidic chamber as used in [21], while more conventional loading systems ${ }^{[3,6]}$ require well-synchronized control of mechanical stretchers. Because mechanical stretchers are not employed, our method can simultaneously handle several systems at the scale of $1 \mathrm{~cm}$ within the film deposition cavity, whose space is often limited. Using this system, we clearly captured transitions from wrinkles to localized ridges and formation of a ridge network surrounding flattened regions. In particular, the experiments reveal the evolution of the ridge structure which involves propagation of individual ridges to form a network. The influence on the geometry of the ridge is explored by varying the pre-stretch and the thickness of the thin film. Further, finite element simulations have been carried out to obtain conditions for localized ridge formation and propagation. Unlike sinusoidal or herringbone wrinkles, the ridge is a finite amplitude mode, and the wrinkle to ridge transition is subcritical as described more fully in conjunction with the simulations. As the pre-stretch is released, once a ridge forms, it grows and saturates to a height comparable to its width.

Figure 1a illustrates the method of preparing the highly pre-stretched substrate. A chamber capped by a flat layer of the elastomeric substrate material, Polydimethylesiloxane (PDMS), is formed. The substrate layer is then stretched by filling the chamber with oil. With the pressure inside the chamber held constant, an organic polymer material, Parylene-C, is deposited on the surface to form the film. Prior to deformation the PDMS substrate layer has a thickness in the range, $h_{s}=50 \mu \mathrm{m}$ to $300 \mu \mathrm{m}$ and a shear modulus, $\mu_{s} \sim 0.33 \mathrm{MPa}$. The Parylene film (thickness $h_{f}$ from $85 \mathrm{~nm}$ to $1.3 \mu \mathrm{m}$ thick, shear modulus $\mu_{f} \sim 0.58 \mathrm{GPa}$ ) is stress-free as deposited. The stress-strain curves of PDMS and Parylene are given in Figure S1, and the detail of the fabrication is available in Supporting Information. The equi-biaxial pre-stretch $\lambda_{0}$ attained in the central region of the substrate is defined as the ratio of the length after stretch $L$ to the original length $L_{0}, \lambda_{0}=L / L_{0}$. An equi-biaxial compressive strain, $\varepsilon$, is exerted on the film in the central region when oil is released incrementally from 
the chamber. The compressive strain is defined as $\varepsilon=1-l / L$ where the current length is $l$. Compared methods such as thermal expansion ${ }^{[4]}$ and swelling ${ }^{[2,22,23,24]}$ for inducing equibiaxial strain, the present method can induce pre-stretch greater than 1.5 and the magnitude of the pre-stretch can be readily adjusted by controlling the pressure inside the chamber. The thickness of the substrate is selected to be at least 50 times that of the film, and the film is about 2000 times stiffer than the substrate. The radius of the chamber is $2 \mathrm{~mm}$, and the effect on the instability due to the curvature of the chamber is considered to be negligible (discussed in Supporting Information). Figures $\mathbf{1 b}$ and $\mathbf{c}$ show the typical morphology of the surface with ridges. Figure $1 \mathrm{~b}$ shows an overview of an experimental setup taken by a microscope at point in the release process where ridges have formed with the chamber still substantially inflated. Figure 1c shows an image of Scanning Electron Microscopy of a surface of another sample. This figure reveals localized ridges separated by extensive unbuckled regions. Unlike a typical wrinkle or herringbone buckling pattern ${ }^{[2,3]}$ which fills the entire surface of the film, the deformation producing a ridge is localized with extensive regions on both sides of the ridge being almost flat. Furthermore, unlike creases and folds ${ }^{[8,9]}$ which protrude into the substrate, ridges push upward from the substrate. These and other aspects of the ridge mode will be discussed in further detail later in the paper. The scale of the ridges depends on the film thickness $h_{f}$. In Figure 1b, with $h_{f} \sim 1 \mu \mathrm{m}$, the width of the ridges is $\sim 50 \mu \mathrm{m}$, while in Figure 1c, with $h_{f} \sim 100 \mathrm{~nm}$, the width of the ridges is $\sim 5 \mu \mathrm{m}$. In neither of these cases has the Parylene thin film delaminated from the PDMS substrate (a cross-sectional view is available in Figure S2). The pre-stretch and compressive strain in the central region are estimated by measuring the height of the chamber at each stage of inflation or deflation, and the non-uniformity of the pre-stretch of the system is considered to be negligible; see Supporting Information (Figure S3) for more details. 
An overview description of one experiment is now presented. As the equi-biaxial compression in the film is increased by slowly releasing oil from chamber, wrinkles first form and then, with further compression, wrinkles give way to ridges as seen in Figure 2. In this experiment, $h_{f}=100 \mathrm{~nm}, h_{s}=50 \mu \mathrm{m}$ and the substrate has been pre-stretched to $\lambda_{0}=1.59$. For this system, dimpled wrinkles, reflecting the equi-biaxial compressive stress experienced by the film, cover the surface at $\varepsilon \cong 0.05$ (Figures $2 \mathbf{a}$ and $\mathbf{b}$ ). As the compressive strain experienced by the film is further increased the dimples become more like wrinkles (Figures 2c) and then, by $\varepsilon=0.066$, the transition to ridge-like features is evident (Figures $\mathbf{2 d}$ and $\mathbf{e}$ ). At $\varepsilon=0.066$, ridges are observed to advance flattening neighboring regions under conditions when no oil is released from the chamber such that the overall compression in the system is nominally fixed (Figure 2f). As will be shown later, the critical compressive strain for ridge formation is only slightly above that for the wrinkle pattern, and in the present study it has not been possible to experimentally resolve the difference between the two critical strains. Further experimental details on the development and evolution of a ridge network are presented in Figure 3. For this experiment, the substrate is pre-stretched to a larger value, $\lambda_{0}=1.85$, enhancing ridge formation and the ensuing ridge network. In this case, $h_{f}=85 \mathrm{~nm}$ and $h_{s}=50 \mu \mathrm{m}$. As shown in Figure 3a, unconnected ridges are first formed with wrinkles appearing perpendicular to the ridges. With the increase of compression, new ridges nucleate and propagate perpendicularly to existing ones until they become blocked by a pre-existing ridge (Figure $\mathbf{3 b}$ and $\mathbf{c}$ ). A network of ridges is created; the few wrinkles still present illustrate clearly the distinction between wrinkles and ridges. With the further increase of compression, the network grows denser (Figure 3d and e) until there is no space for new ridges to be nucleated and the network saturates (Figure 3f). The evolution seen here for the ridge pattern is similar to the evolution of folds in a compressive bi-layer without pre-stretch reported in [8]. 
The dependence of the geometry of the ridge and the threshold pre-stretch $\lambda_{c}$ required for the formation of a ridge has been measured by systems with seven different thicknesses of the Parylene film ranging from $h_{f}=85 \mathrm{~nm}$ (with $h_{s}=50 \mu \mathrm{m}$ ) to $h_{f}=1.3 \mu \mathrm{m}$ (with $h_{s}=300 \mu \mathrm{m}$ ). For a given pre-stretch, $\lambda_{0}$, the compressive strain $\varepsilon$ is increased by release of the pre-stretch and the surface of the bi-layer is carefully examined to see the buckling patterns that emerge and, in particular, whether ridges form. The phase diagram of wrinkle/ridge is plotted in Figure 3g. The threshold pre-stretch is approximately $\lambda_{c}=1.4$ for ridge formation-that is, for smaller pre-stretches ridges are not observed and for larger pre-stretches they are observed. The threshold appears to be independent of the thickness of the stiff film, consistent with what is expected on dimensional grounds for thick substrates. For cases in which ridges are formed, the width $w$ and the height $A$ of the ridges (as defined in the inset of Figure $3 \mathrm{~h}$ ) are also measured and plotted in Figure 3g. The measurements presented in the Figure $3 \mathrm{~h}$ have been taken for cases in Figure $3 \mathrm{~g}$ in which the pre-stretch $\lambda_{0}$ is between 1.4 and 1.6. The width $w$ is proportional to the film thickness $h_{f}$, and for the stiffness ratio of the present film/substrate system the experimental results are fitted by $w=34 h_{f}$. If one imagines a ridge to be the localization of a single wrinkle, one would expect the ridge width $w$ to be the same order as the wavelength of the wrinkle $l_{w}$. In [10], the wavelength of a wrinkled linear elastic film on a neo-Hookean substrate subject to an equi-biaxial pre-stretch $\lambda_{0}$ is $l_{w}=2 \pi h_{f}\left(2 \mu_{f} \lambda_{0} /\left(3 \mu_{s}\left(1+\lambda_{0}^{3}\right)\right)\right)^{1 / 3}$. With $\mu_{f} / \mu_{s}=1750$ and $\lambda_{0}=1.5$, one finds $l_{w}=46 h_{f}$, which is on the same order of the experimentally measured wavelength in Figure $3 \mathrm{~h}$. In all of these cases, the ratio of the ridge height $A$ to its width $w, A / w$ (inset of Figure $3 h$ ) is significantly larger than the amplitude/wavelength ratio of the conventional wrinkle at these compressive strains, i.e., $\sim 0.1$. 
Numerical simulations employing the commercial finite element software, ABAQUS, have been carried out to study and quantify the conditions for ridge formation. These simulations go well beyond the initial plane strain studies ${ }^{[10]}$ where ridges were first discovered theoretically. In addition to systematically exploring the effect of equi-biaxial pre-stretch on ridge formation, we have investigated the mechanics by which the ridges propagate. Simulation details can be found in Supporting Information, and a detailed paper on the mechanics of ridge formation and propagation will be published at a later date. To break the translational symmetry of the flat surface and initiate wrinkles, a very small initial imperfection in the form of a slight surface waviness is introduced. The formation of wrinkles and ridges is simulated under a generalized plane strain condition in the 1-2 plane (as defined in Figure 4a) with an equi-biaxial pre-stretch $\lambda_{0}$ in the substrate and the subsequent equibiaxial compressive strain $\varepsilon$ applied to both film and substrate. In keeping with generalized plane strain, the overall strains in the 1-3 directions are equi-biaxial, but the displacements caused by the wrinkles or by ridges relative to the equi-biaxial state are subject to the constraint of plane strain. The plane strain constraint limits wrinkling and ridging to onedimensional entities - the dimple mode seen in Figure 2 and the 2D ridge pattern in Figure 3 are excluded. Nevertheless, the essence of ridge formation is captured within the constraint adopted. Both film and substrate are modeled as incompressible neo-Hookean materials. Ridges grow at the expense of neighboring wrinkles and, therefore, to simulate ridge localization the width of the computational model must be at least several times the wrinkle wavelength. In the present simulation, this width is $L \cong 10 l_{w}$. By making use of symmetry, only half of this length is simulated and, as shown in Figure 4a, the symmetric condition is set at the left boundary.

When $\varepsilon$ reaches a critical strain denoted by $\varepsilon_{w}$, wrinkles start to form in the 1-direction with wavelength $l_{w}$. The computed values of $\varepsilon_{w}$ and $l_{w}$ are in close agreement with predictions of 
the classical bifurcation results for wrinkling ${ }^{[10]}$. As the applied compression is further increased, the wrinkle amplitudes increase but the nominal wrinkle wavelength does not change.

We have found that the wrinkle to ridge transition is unstable (subcritical in the language of bifurcation theory) such that a wrinkle snaps dynamically to a ridge with a much larger amplitude. Conventional quasi-static simulations fail for such phenomena and special numerical techniques are required, such as the arc-length method ${ }^{[25,26]}$ or dynamic simulations. We have developed the following quasi-static technique to probe for the existence of a ridge and found it to be systematic and reliable. As depicted in Figure 5a, under the fixed overall strain $\varepsilon$, an additional vertical displacement $\Delta$ is imposed on the peak of the wrinkle at the left boundary. The associated reaction force $F(\Delta)$ is computed. The system remains stable when the additional vertical displacement $\Delta$ is prescribed, and this allows large displacement equilibrium states to be discovered wherever $F=0$. If a state with $F=0$ is found when $\Delta$ is non-zero, then that state can exist (without an external force) at the strain $\varepsilon$ although it may be stable or unstable. An example of this process is shown in Figure $\mathbf{4 b}$. The ridge seen in Figure $4 \mathrm{~b}$ (at $F=0$ ) is clearly a localized mode in sense that the amplitudes of the wrinkles neighboring it have diminished significantly.

Figures 4c-e show the $F-\Delta$ relation under different fixed strains $\varepsilon$ for three pre-stretches, $\lambda_{0}=1.2,1.4$, and 1.6, in the substrate. The shear modulus ratio of the film to the substrate $\mu_{f} / \mu_{s}$ has been taken to be 2000 , slightly larger than that of the experimental system for simplicity, and the thickness ratio of the bi-layer to the film is set to be 50 so that the substrate can be considered much thicker than the film. When $\lambda_{0}=1.2$, the critical condition for wrinkle initiation is approximately $\varepsilon_{w} \cong 0.0022$. As shown in Figure $4 \mathrm{c}$, when the strain $\varepsilon$ is fixed at $0.016, F$ sharply increases with $\Delta$ and $F$ does not become zero. In fact, for all of the fixed strains $\varepsilon$, from 0.016 to 0.022 , the $F-\Delta$ curves almost overlap. The behavior for a 
larger pre-stretch, $\lambda_{0}=1.6$, is dramatically different. For small applied compression, $\varepsilon=0.008$, $F$ increases monotonically with $\Delta$ (Figure 4e). However, for larger $\varepsilon$, the $F-\Delta$ curves become non-monotonic: $F$ increases, decreases and then increases again with $\Delta$. Above a critical $\varepsilon$, the $F-\Delta$ curve intersects the $\Delta$-axis at three points: the origin, which represents the wrinkle state, the middle point, which represents an unstable medium amplitude ridge, and the point on the right, which represents a stable large amplitude ridge. This plot clearly shows the large amplitude nature of ridges. Figure $4 \mathrm{a}$ shows the simulation result of wrinkles for $\varepsilon=0.011$ when $\lambda_{0}=1.6$. Figure $4 \mathrm{~b}$ shows the simulation result for the stable large amplitude ridge for the same strain and pre-stretch (as indicated by a circle in Figure 4e). The additional film length needed to form the ridge is obtained by flattening neighboring wrinkles, and it is this feature which gives rise to localization. At the intermediate pre-stretch, $\lambda_{0}=1.4$, the $F-\Delta$ relation is strongly nonlinear. However, even at $\varepsilon=0.03$ (not shown in Figure 4 d), $F$ does not become zero at non-zero $\Delta$. To conclude, our simulations suggest that the threshold value of pre-stretch for ridges to form is slightly larger than $\lambda_{0}=1.4$. This is in general agreement with the experimental observations reported in Figure 3g.

Additional simulations have been performed to show the evolution of the ridges by a pseudo-dynamic simulation (Figure 5). Again, an initial imperfection consisting of a slight waviness is introduced to trigger the initiation of the wrinkles. The bi-layer is loaded equibiaxially with an equi-biaxial pre-stretch $\lambda_{0}=1.6$ in the substrate. The modulus ratio and thickness ratio are the same as Figure 4. With a gradual compression increase, well defined wrinkles first form. When the compression $\varepsilon$ increases to about 0.0185 , a ridge initiates and snaps to a state with finite amplitude. As noted earlier, the ridge forms at the expense of neighboring wrinkles. The ridge quickly grows to a saturated height when $\varepsilon$ reaches around 0.0205. With the further increase of compression, more ridges form. However, the height of previously formed ridges changes relatively little with increasing compression consistent with 
the notion of a saturated height. For $\varepsilon=0.1163$, the ratio of the height of the ridges to the film thickness predicted for this system is around $A / h_{f}=24$, and the ratio of the width of the ridges to the nominal film thickness is around $w / h_{f}=29$, such that $A / w=0.83$. These characteristic length scales of the ridges and the evolution of their behavior predicted by simulations are in reasonable general agreement with the experiments. The experimental measured width to film thickness ratio in Figure $3 \mathrm{~h}$ is about $15 \%$ higher than the prediction. While the theoretical height to thickness ratio is within the range of the experimental results in Figure $3 \mathrm{~h}$, the experimental measurements display considerably more variation. In part, this can be explained by the fact that there is some dependence of the ridge amplitude on the applied strain after it is formed. In addition, there may be some influence of the finite thickness of the substrate on $A / W$, which is not accounted for in the numerical simulations, because the height of the ridge is comparable to the substrate thickness in the experiment. The main discrepancy between simulation and experiment is the critical strain for the initiation of wrinkles and ridges - simulations predict somewhat lower critical strains than those measured experimentally. In previous work ${ }^{[27]}$, residual stress in Parylene film has been noted. For the present system, the residual tensile strain in the Parylene film on PDMS substrate has been measured as $\sim 1 \%$ (Figure S4). The applied compression $\varepsilon$ has to overcome any residual tensile strain in the film, and it is believed that this accounts for the discrepancy mentioned above.

To summarize, the evolution of the buckling patterns of bi-layer film-substrate systems has been studied for systems having a large equi-biaxial pre-stretch in the substrate. A microfluidics chamber has been designed for carrying out the experiments. The pre-stretch in the substrate is tuned by varying the volume of the oil in the PDMS chamber, and the compressive strain is applied to the bi-layer system by diminishing the volume of oil. Transitions from wrinkles to ridges and the formation and evolution of ridge networks are 
clearly captured. Ridges are a highly localized deformation mode resulting in flattening of the film in its vicinity. Details of ridge formation and geometry have been studied by varying the pre-stretch and the thickness of the thin film. Finite element simulations have been carried out to reveal the highly nonlinear character of ridges and to quantify conditions for their formation. The wrinkle to ridge transition is unstable, or subcritical, and the ridge amplitudes are much larger than those of the wrinkles. The simulations qualitatively capture the experimental results in all respects and reproduce most aspects quantitatively with reasonable success. The ability of the present system to attain high pre-stretch enriches the scope for exploring surface instability patterns of bi-layer systems. The ridge mode has higher aspect ratios than the wrinkle mode and a ridge network consists of isolated patches of flat surface surrounded by ridges. It is hoped that our method will open a new way to create and improve the properties of functional surfaces, such as hydrophobicity, adhesion and micro-patterning.

\section{Experimental}

Surface transition and ridge evolution: In the experiment in Figure 2, the compressive strain is slowly applied by reducing the oil at the rate of $1 \mu \mathrm{l} / \mathrm{min}$ from the PDMS chamber. The surface is observed with a conventional optical microscope. The focal plane of the microscope is set at the summit of the chamber during the process. For the experiment in Figure 3, the surface is observed with an optical surface measurement system which contains a microscope and a laser profiler (Keyence, VK-8710). Image processing software in the system improves the optical images by cancelling the gap of the focal plane induced by the curvature.

\section{Acknowledgements}

We acknowledge Prof Yanping Cao at Tsinghua University for a discussion on simulating ridges. This work was partially supported by Grant-in-Aid for JSPS Fellows. 
[1] C. M. Stafford, C. Harrison, K. L. Beers, A. Karim, E. J. Amis, M. R. Vanlandingham, H. C. Kim, W. Volksen, R. D. Miller, E. E. Simonyi, Nature Materials, 2004, 3, 545.

[2] S. Cai, D. Breid, A. J. Crosby, Z. Suo, J. W. Hutchinson, Journal of the Mechanics and Physics of Solids, 2011, 59, 1094.

[3] P. C. Lin, S. Yang, Applied Physics Letters, 2007, 90, 241903.

[4] N. Bowden, S. Brittain, A. G. Evans, J. W. Hutchinson, G. M. Whitesides, Nature, 1998, 393, 146.

[5] Q. Wang, X. Zhao, Journal of Applied Mechanics, doi:10.1115/1.4025828

[6] Changyong Cao, Hon Fai Chan, Jianfeng Zang, Kam W. Leong, Xuanhe Zhao, Advanced Materials, In press

[7] F. Brau, H. Vandeparre, A. Sabbah, C. Poulard, A.Boudaoud, P. Damman,Nature Physics, 2011, 7, 56.

[8] L. Pocivavsek, R. Dellsy, A. Kern, S. Johnson, B. Lin, K.Yee, E. Cerda, Science, 2008, 320, 912.

[9] P. Kim, M. Abkarian, H. A. Stone, Nature Materials, 2011,10, 952.

[10] Y. Cao, J. W. Hutchinson, Journal of Applied Mechanics, 2012. 79, 031019-1

[11] Y. Ebata, A. B. Croll, A. J. Crosby, Soft matter, 2012, 8, 9086.

[12] E. P. Chan, E. J. Smith, R. C. Hayward, A. J. Crosby, Advanced Materials, 2008, 20, 711.

[13] J. Y. Chung, J. P. Youngblood, C. M. Stafford, Soft Matter, 2007, 3, 1163

[14] P. C. Lin, S. Yang, Soft Matter, 2009, 5, 1011.

[15] S. G. Lee, H. S. Lim, D. Y. Lee, D. Kwak, K. Cho, Advanced Functional Materials, 2012, 23, 547.

[16] C. Badre, J. P. Chapel, S. Yang, Soft Matter, 2011, 7, 9886.

[17] J. Zang, X. Zhao, Y. Cao, J. W. Hutchinson, Journal of the Mechanics and Physics of Solids, 2012, 60, 1265.

[18] D. Vella, J. Bico, A. Boudaoud, B. Roman and P. M. Reis, Proceedings of the National Academy of Sciences of the United States of America, 2009,106, 10901

[19] J. Zang, S. Ryu, N. Pugno, Q. Wang, Q. Tu, M. J. Buehler and X. Zhao, Nature Materials, 2013,12, 321

[20] A. L. Volynskii, S. Bazhenov, O. V. Lebedeva and N. F. Bakeev, Journal of materials science, 2000, 35, 547

[21] M. Staykova, D. P. Holmes, C. Read, H. A. Stone, Proceedings of the National Academy of Sciences of the United States of America, 2011,108, 9084

[22] M. Guvendiren, S. Yang, J. A. Burdick, Advanced Functional Materials, 2009. 19, 3038

[23] D. Breid, A. J. Crosby, Soft matter, 2011, 7, 4490.

[24] D. Breid, A. J. Crosby, Soft matter, 2013, 9, 3624.

[25] E. Riks, Journal of Applied Mechanics, 1972, 39, 263.

[26] D. Chen, L. Jin, Z. Suo, R. C. Hayward, Materials Horizons, 2014 DOI: $10.1039 / \mathrm{C} 3 \mathrm{MH} 00107 \mathrm{E}$

[27] S. Dabral, J. Van Etten, X. Zhang, C. Apblette, G. Yang, P. Ficalora, J. McDonald, Journal of Electronic Materials, 1992, 21, 989 

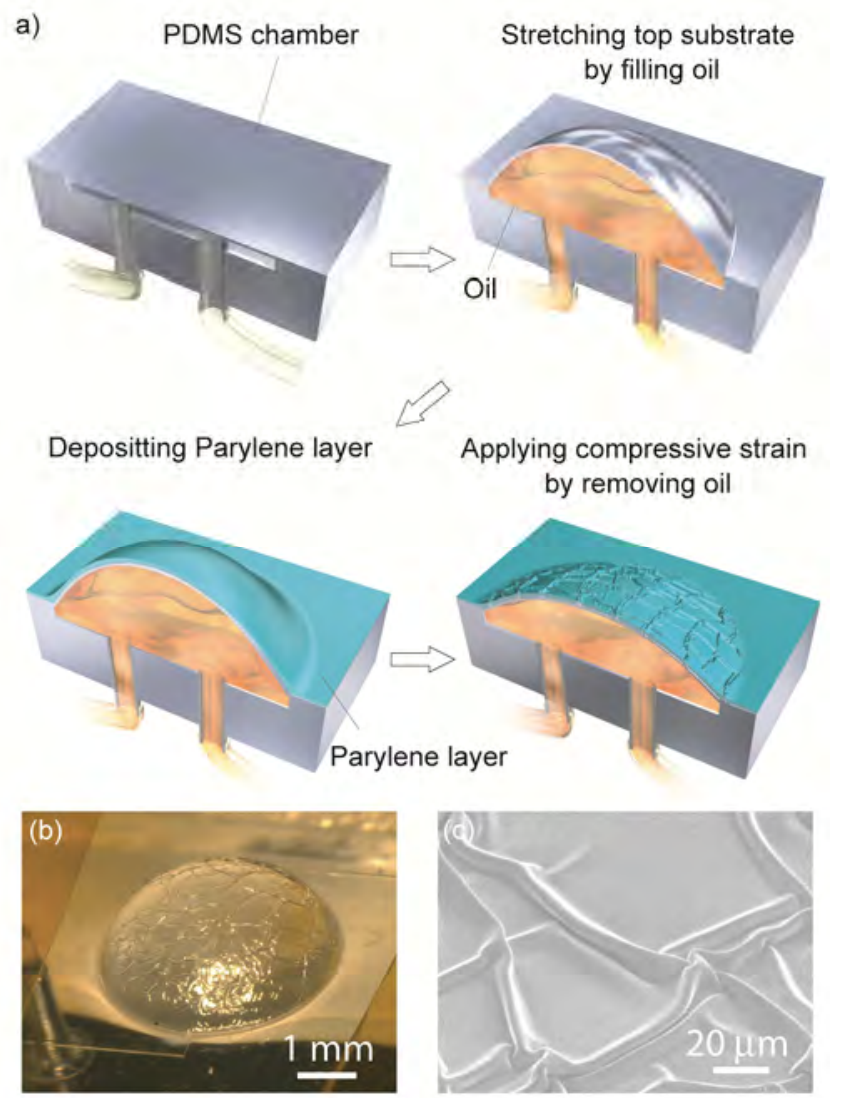

Figure 1. (a) Schematic illustration showing the preparation of the highly pre-stretched bilayer system using micro-fluidics. (b) Image of the experimental setup: Compressive strain is applied by reducing the oil inside the chamber and a ridge structure emerges on the surface. (c) SEM image of the ridge structure: the localized convex structure is observed concomitantly with a flattening of the neighboring film surface. 


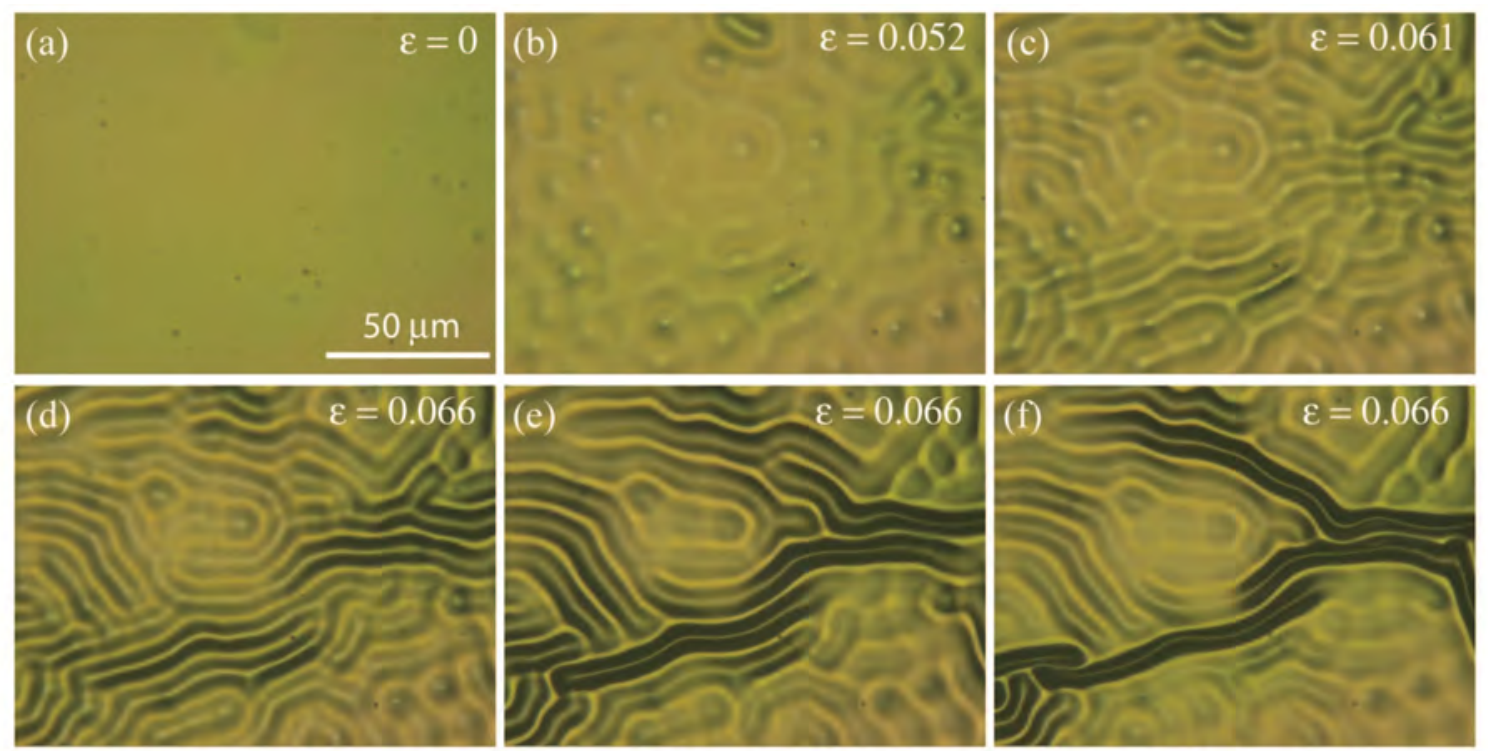

Figure 2. Wrinkle to ridge transition. (a) In its initial state the surface of the chamber is flat. (b) With compressive strain, the dimpled wrinkle pattern is engendered on the surface. (c) With increase of the compressive strain, the dimpled wrinkle pattern transforms into the wrinkle pattern. (d) With further compression, some peaks of the wrinkles increase their height and ridges are nucleated. (e, f) Under essentially the same applied compression, ridges can propagate and flatten neighboring surfaces. 

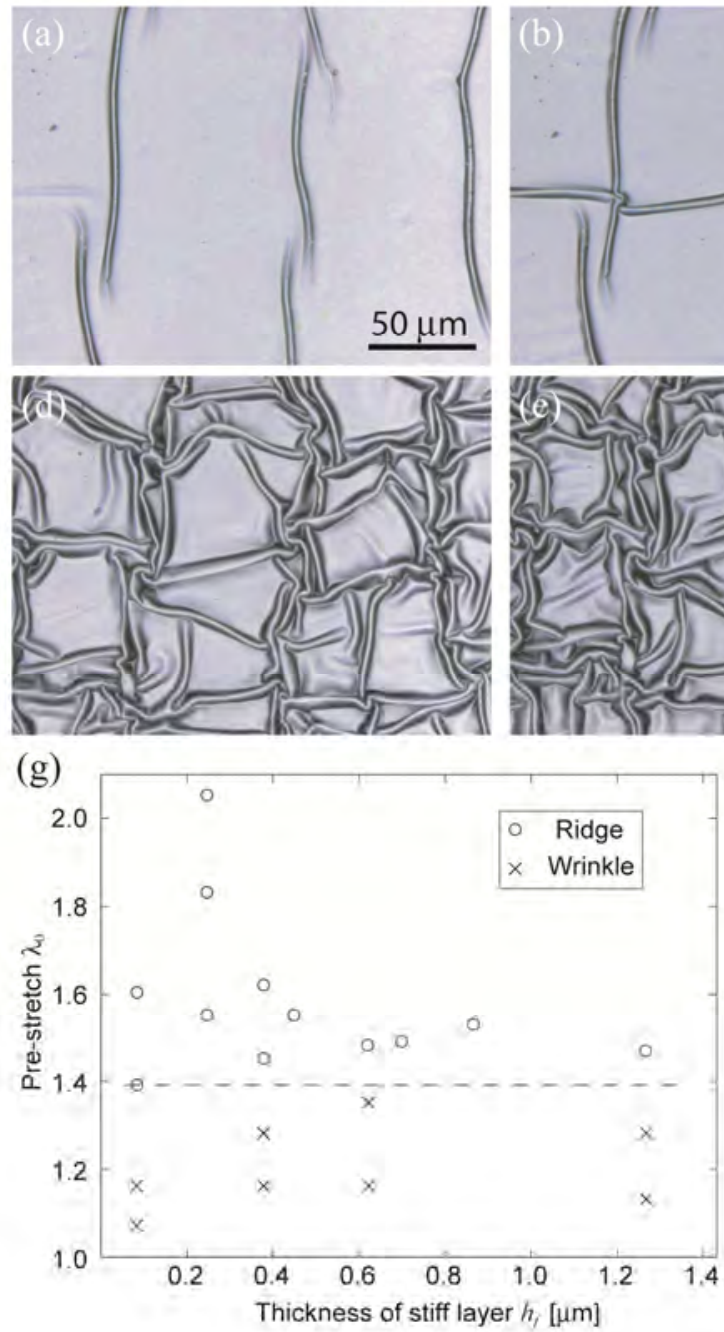
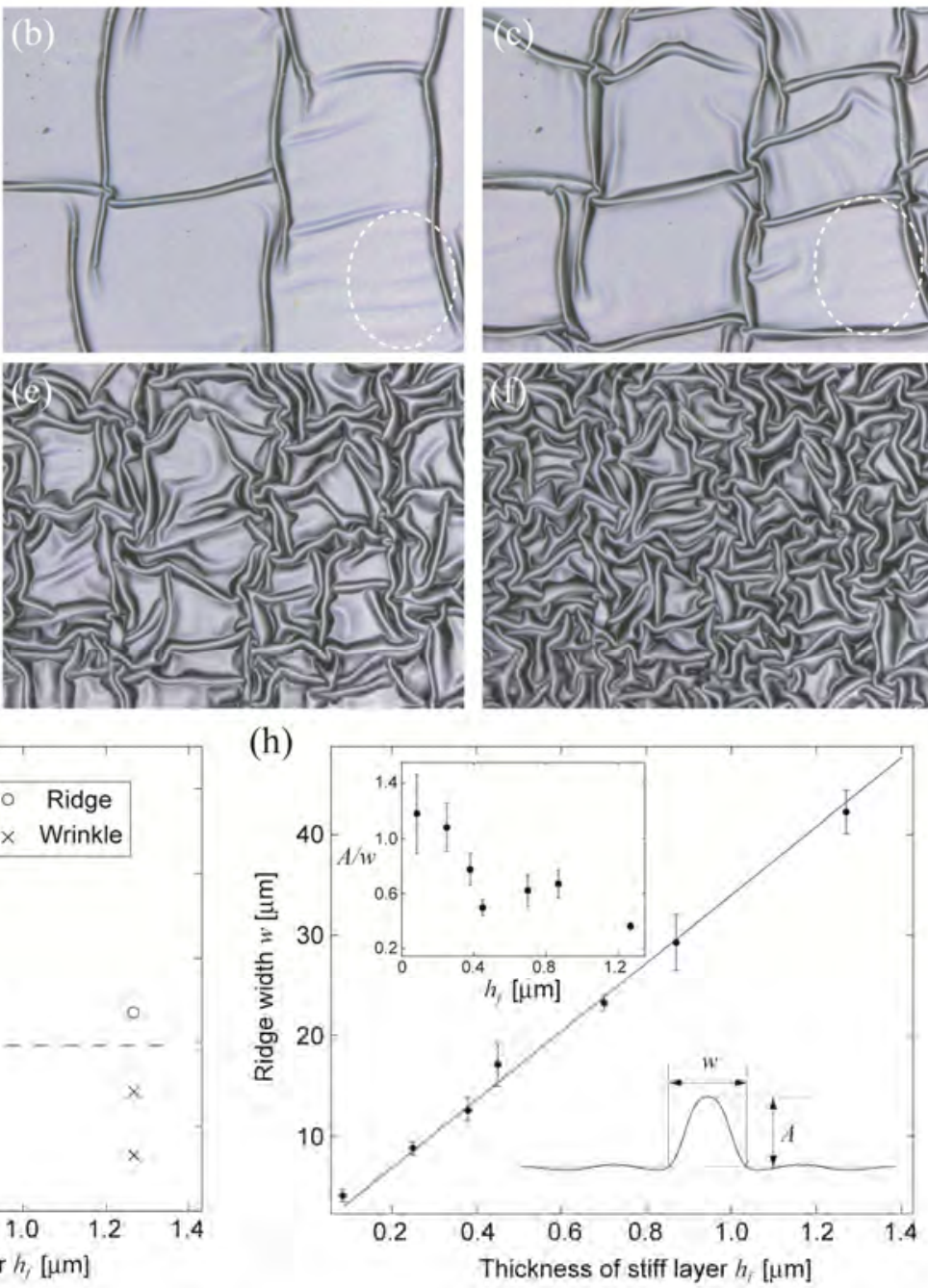

Figure 3. The formation of the ridge network: (a) In compression, unconnected ridges first appear. (b-c) With the further increase of the compression, new ridges nucleate perpendicular to the existing ridges. As marked with the dashed circles, wrinkled regions emerge before ridge nucleation and become flattened after the formation of the ridge. (d-e) With even further increase of the compression, the network grows denser, and, (f), finally is completely packed. (g) Phase diagram of wrinkle/ridge mode. Ridge structure appears with the pre-stretch $\lambda_{0}>1.4$. (h) Relationship between stiff film thickness and width of the ridge. Fitting curve is $w=34 h_{f}$. Relationship between height/width ratio $A / w$ of a ridge and stiff film thickness $h_{f}$ is embedded. 
(a)

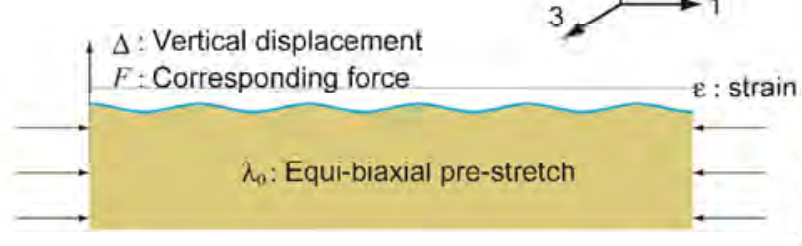

(b)

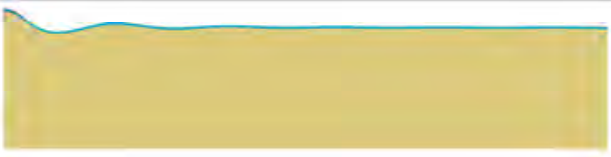

(d) $\lambda_{0}=1.4$

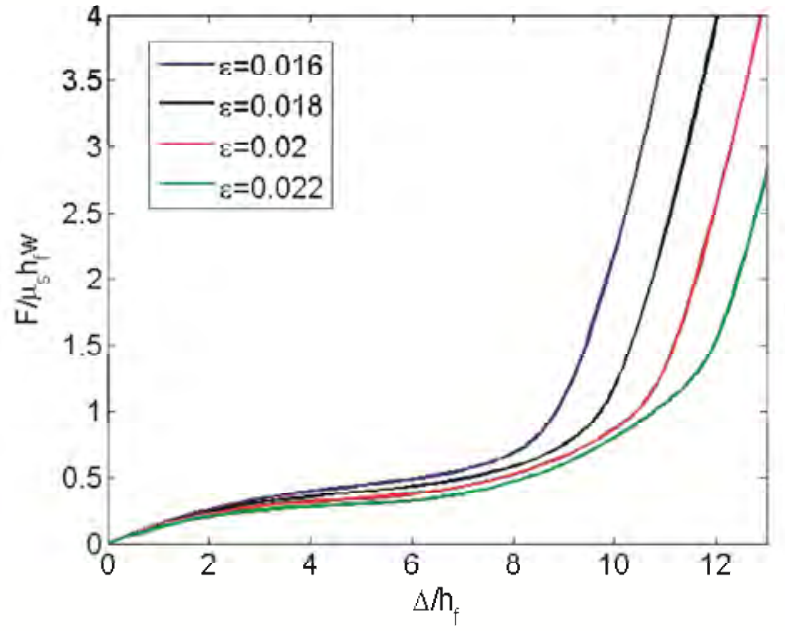

(c) $\lambda_{01}=1.2$

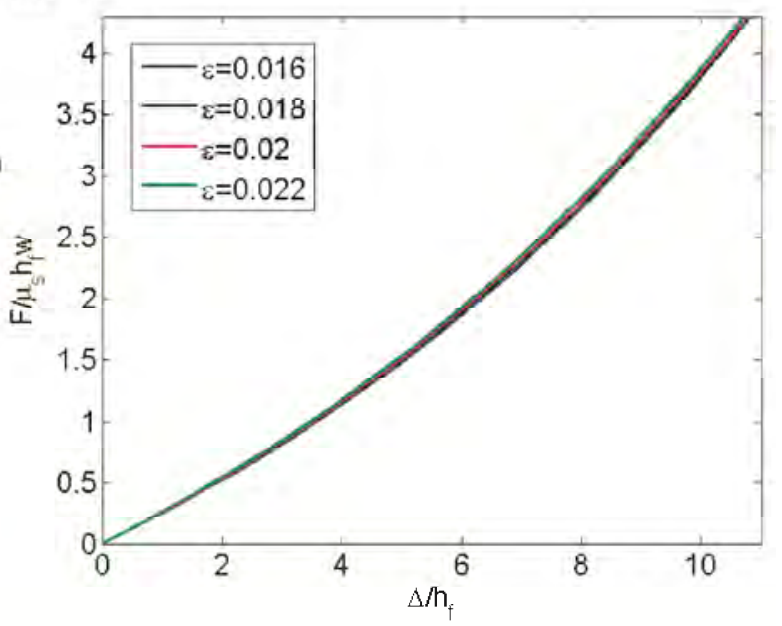

(e) $\lambda_{0}=1.6$

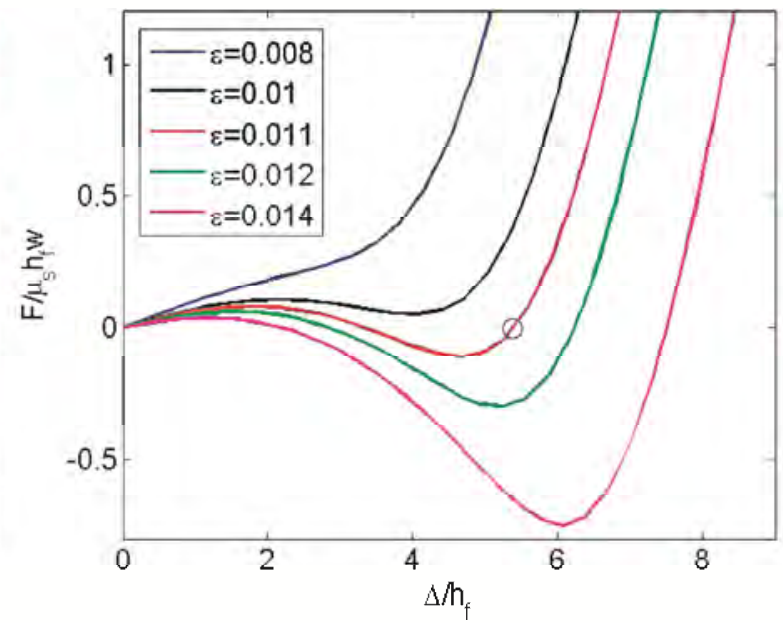

Figure 4. Finite element simulation results. (a) is a simulation result showing wrinkles under equi-biaxial strain $\varepsilon=0.011$ and pre-stretch $\lambda_{0}=1.6$ in the substrate. (b) is a simulation result showing the large amplitude ridge state under the same loading condition as (a). In (a) and (b), the blue color shows the thin film and the yellow brown color shows the substrate. The $F-\Delta$ curves are obtained for various fixed strains for equi-biaxial pre-stretch (c) $\lambda_{0}=1.2$, (d) $\lambda_{0}=1.4$, and (e) $\lambda_{0}=1.6$ with $\mu_{f} / \mu_{s}=2000$ and $\left(h_{f}+h_{s}\right) / h_{f}=50$. 


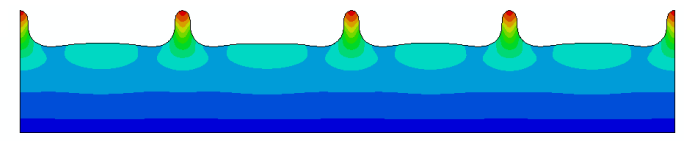

$\varepsilon=0.0141$

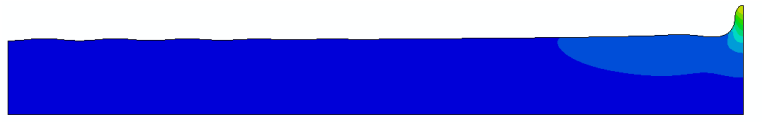

$\varepsilon=0.0205$

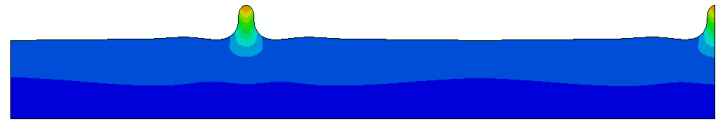

$\varepsilon=0.0597$

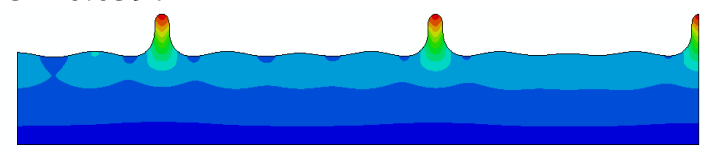

$\varepsilon=0.1163$

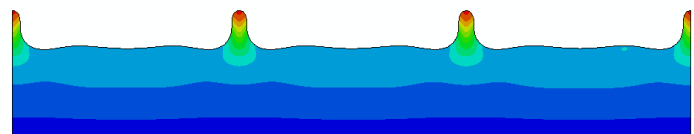

$\varepsilon=0.1289$ $\varepsilon=0.1587$

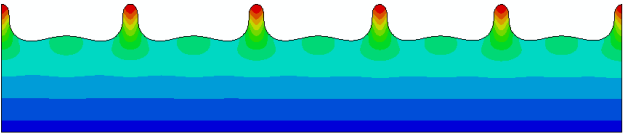

$\mathcal{E}=0.1940$

Figure 5. Evolution of the morphology of the film-substrate bi-layer at different equi-biaxial strains $\varepsilon$ revealing the progression to wrinkles and then to ridges. The color bar represents the normalized vertical displacement $U_{2} /\left(h_{f}+h_{s}\right)$. The parameters used in the simulation are as followings: $\lambda_{0}=1.6, \mu_{f} / \mu_{s}=2000$, and $\left(h_{f}+h_{s}\right) / h_{f}=50$. 


\section{Supporting Information}

Ridge localizations and networks in thin films compressed by incremental release of large equi-biaxial substrate pre-stretch

Atsushi Takei, Lihua Jin, John. W. Hutchinson, and Hiroyuki Fujita

Contents

1. Experimental details

2. Figure S1. Stress-strain curves of PDMS and Parylene

3. Figure S2. Cross sectional view of ridge structure

4. Figure S3. Pre-stretch and compressive strain estimation by finite element simulation

5. Figure S4. Estimation of residual stress in Parylene membrane 


\section{Experimental Details}

PDMS chamber fabrication: $50 \mu \mathrm{m}$ or $300 \mu \mathrm{m}$ thick PDMS (Dow corning, Sylgard 184) substrate is spin coated on a glass substrate and cured for 2 hours at $75^{\circ} \mathrm{C}$. A PDMS microfluidics structure is also prepared using a silicon mold which has a concave circular part with a $2 \mathrm{~mm}$ diameter and holes for an inlet and outlet. The two components are bonded by applying an oxygen plasma (20W, $100 \mathrm{sccm}, 20 \mathrm{~Pa}, 30$ second) to both surfaces. The cross linker/base ratio of PDMS is $1: 10$, and its shear modulus is $0.33 \mathrm{MPa}$. The PDMS chamber is inflated by filling Silicone oil (Shinetsu, HI-VAC-F-4), which is degassed for at least 2 hours before film deposition. The Parylene membrane is then deposited on the surface with a lab coater (Speciality Coating Systems, PDS2010). The shear modulus of Parylene is $\sim 0.58$ GPa. Because it is difficult to measure the thickness of the film deposited on the hemispherical PDMS substrate, a glass substrate is also put into the deposition chamber and the Parylene layer is simultaneously deposited on the glass substrate under identical conditions. The thickness of the Parylene layer, $h_{f}$, is measured using the film on the glass substrate with a stylus profiler (KLA Tencor, D-120). The pre-stretch $\lambda_{0}$ in the central region of the substrate is estimated from the shape of the inflated chamber. The relation between the pre-stretch $\lambda_{0}$ and the shape is calculated using the commercial software, ABAQUS (See, supplementary info). The initial height of the chamber in the experiments in Figure 2 and 3 is $1.71 \mathrm{~mm}$ and $2.02 \mathrm{~mm}$, which corresponds to the pre-stretches $\lambda_{0}=1.59$ and 1.85 in the central region of the bi-layer.

Ridge geometry: In the experiment in Figure 4b, the geometry of the ridge is measured by the same laser profiler used for Figure 3. The measured thicknesses of the Parylene film are $85 \mathrm{~nm}$, $250 \mathrm{~nm}, 380 \mathrm{~nm}, 450 \mathrm{~nm}, 700 \mathrm{~nm}, 870 \mathrm{~nm}$ and $1.3 \mu \mathrm{m}$. For the sample with the $1.3 \mu \mathrm{m}$ film, the thickness of the PDMS substrate is $300 \mu \mathrm{m}$; for all the other samples, the thickness of the 
substrate is $50 \mu \mathrm{m}$. According to Cai et al ${ }^{[2]}$, if the Poisson's ratios of the both material $v$ are identical, the effect of the radius of curvature of the chamber, $R$, on wrinkling is quantified by the following dimensionless parameter:

$$
\Omega=2 \sqrt{1-v^{2}}\left(h_{f} / R\right)\left(\mu_{f} / 3 \mu_{s}\right)^{2 / 3}
$$

In the present experimental set up, the maximum value of $\Omega$ is $\sim 0.1$ at $h_{f}=1.3 \mu \mathrm{m}, v=0.5$ and $R=2.0 \mathrm{~mm}$. Here, $R$ is considered to be of the same order of the radius of the chamber. This small value $\Omega$ implies that curvature of surface of the bi-layer has essentially no effect on the wrinkling and ridging behavior.

Finite element simulations: The finite element software, ABAQUS, was used to study the formation and growth of ridges. For both simulations shown in Figures 5 and 6, a linear perturbation simulation is first carried out to identify the critical strain of wrinkle initiation $\varepsilon_{w}$ and the critical wavelength $l_{w}$. Then, the critical wrinkle mode with wavelength $l_{w}$ times a very small amplitude factor ( 0.0001 of the total thickness of the film and substrate) is introduced as the initial imperfection to break the translational symmetry for the simulations in Figure 5 and 6. Both the film and substrate are modeled as incompressible neo-Hookean materials, with shear modulus ratio of the film to substrate, $\mu_{f} / \mu_{s}=2000$, and thickness ratio of the bi-layer to film $\left(h_{f}+h_{s}\right) / h_{f}=50$. In Figures 5 and 6, the equi-biaxial compression of the bi-layer structure is simulated beginning with an equi-biaxial pre-stretch in the substrate. The formation of wrinkles and/or ridges is simulated under generalized plane strain, such that in any increment of loading, a 2D plane strain computation is made (in 1-2 plane as defined in Figure 5a) with invariance in the 3-direction. Overall equi-biaxial compression in the 1- and 3-direction is achieved incrementally in each loading step with the aid of a user-defined material subroutine. With an increment of the applied compressive strain $\varepsilon$ in the 1-direction, the compression in the 3-direction is programmed to coincide with that in 1-direction. Equi- 
biaxial pre-stretch is also implemented in the user-defined material subroutine. In Figure 5, the length of the simulation region is $L / h_{f}=250$ for all pre-stretches, $\lambda_{0}=1.2,1.4$, and 1.6. In Figure 6, the length of the simulation region is $L / h_{f}=500$. In Figure 5, the method which prescribes the vertical displacement at the ridge peak was used, as described above. For Figure 6, a pseudo-dynamic simulation method with numerical stabilization was employed to capture the snap-through process from wrinkles to ridges, with the damping factor set as 0.0002. In addition, a structured mesh technique was used. 


\section{Figure S1. Stress-strain curves of PDMS and Parylene}

Stress-strain curves of PDMS (Figure S1a) and Parylene (Figure S1b) were measured with a force gauge (Instron, Instron 5865), which prescribes displacement and measures load. A PDMS strip with a width of $10 \mathrm{~mm}$ and a thickness of $300 \mu \mathrm{m}$ and a length of $30 \mathrm{~mm}$ was used to obtain the stress-strain curve of PDMS. To obtain the stress-strain curve of Parylene, a Parylene film of $2.4 \mu \mathrm{m}$ thickness was deposited on the PDMS strip, and then the stress-strain curve of the Parylene/PDMS bi-layer system was measured. By using the bi-layer system, plastic necking and cracking of the Parylene film can be avoided. By subtracting the contribution of the PDMS stiffness from the strain-stress curve of the bi-layer system, the stress-strain curve of Parylene was obtained. From the curves, the Young's modulus of PDMS $E_{s}$ and Parylene $E_{f}$ are $1 \mathrm{MPa}$ and $1.75 \mathrm{GPa}$, respectively. Considering both materials to be incompressible (Poisson's ratio $v=0.5$ ), one finds the shear moduli of PDMS $\mu_{s}$ and Parylene $\mu_{f}$ to be $0.33 \mathrm{MPa}$ and $0.58 \mathrm{GPa}$, respectively.
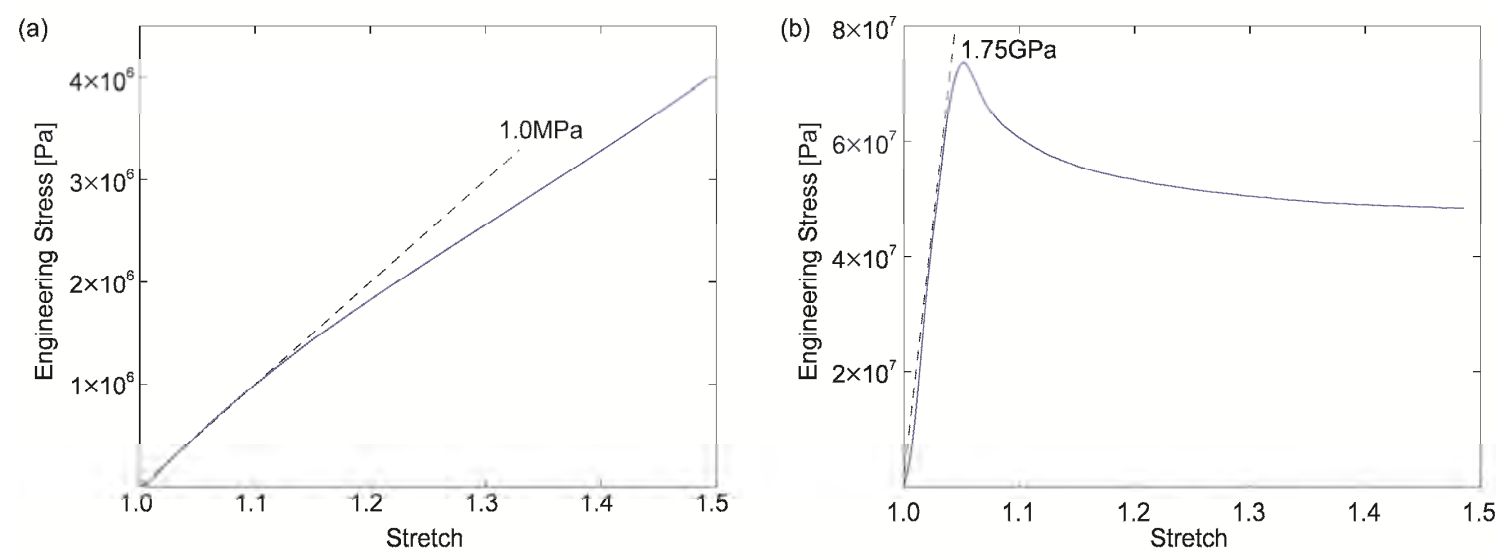


\section{Figure S2. Cross sectional view}

Liquid PDMS is filled in PDMS chamber, then Parylene film ( 800 nm thick) is deposited on the surface. After the deposition, the liquid PDMS is slightly removed to induce the compressive strain. We leave the sample for 1 day at room temperature for solidification of the liquid PDMS, and the sample is cut with a razor. The cross section is observed with SEM. The Parylene film is not delaminated from the PDMS substrate, and the height and the width of the ridge is on the same order. The cross section of Parylene film is highlighted with red line in the right image.
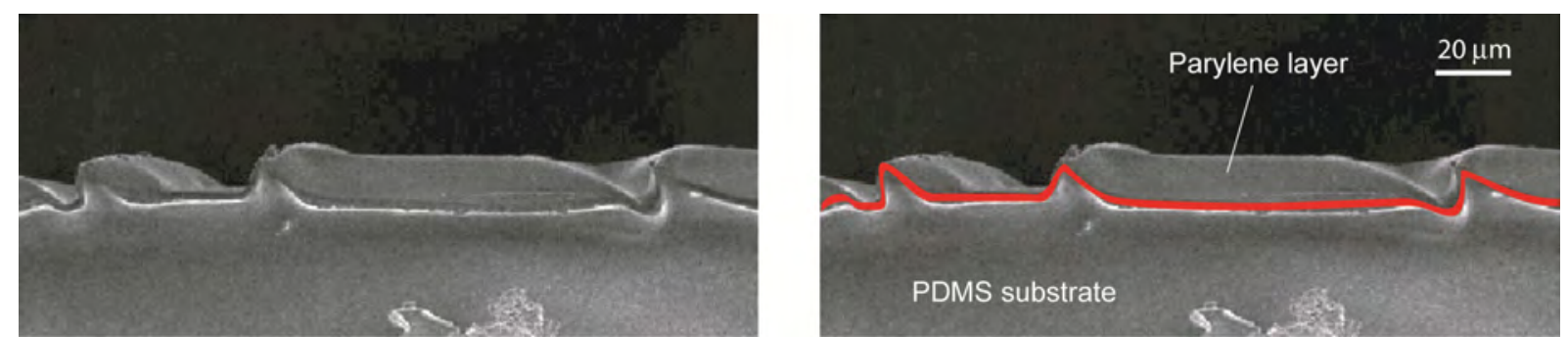


\section{Figure S3. Pre-stretch and compressive strain estimation by finite element simulations}

We use the finite element software, ABAQUS, to relate the pre-stretch $\lambda_{0}$, compressive applied strain $\varepsilon$ in the central region of the substrate to the PDMS chamber height, which is measured in the experiment. An axisymmetric simulation of a PDMS substrate with thickness $50 \mu m$ and radius $2 \mathrm{~mm}$ under pressure inflation is run to calculate the relation of the stretch $\lambda$ in the middle of the chamber as a function of the height $H$ (Figure S3a). In the experiment of Figure 2, the height of the chamber before the deposition of Parylene film is $H_{0}=1.71 \mathrm{~mm}$, which corresponds to the pre-stretch $\lambda_{0}=1.59$. The shape of the chamber and vertical displacement $U_{2}$ is shown in Figure S3c. During the deflation of the PDMS chamber, the thickness of the Parylene is so small that it does not contribute significantly to the bending stiffness and the shape of the chamber. Therefore, the film is not accounted for in the simulation. The compressive strain $\varepsilon$ is $\varepsilon(H)=1-\lambda(H) / \lambda_{0}$, and the $\varepsilon-H$ relation is shown in Figure S3b. The chamber height in Figure $2 \mathrm{a}-\mathrm{d}$ is $1.71 \mathrm{~mm}, 1.60 \mathrm{~mm}, 1.58 \mathrm{~mm}$ and 1.57 $\mathrm{mm}$, which corresponds to $\mathcal{E}=0,0.052,0.061$ and 0.066 respectively. In the experiment of Figure 4b, the initial height of the PDMS chamber is set to be between $1.5 \mathrm{~mm}$ and $1.7 \mathrm{~mm}$, which corresponds to the pre-stretch $\lambda_{0}$ between 1.43 and 1.58 . The central regions of the membrane in which wrinkles and ridges are measured typically have a radius of $100 \mu \mathrm{m}$ for Figure 2 and Figure 3a-f and of $500 \mu \mathrm{m}$ for Figure $3 \mathrm{~g}$ and h. Within these regions there are less than $0.2 \%$ and $2.3 \%$ variation in pre-stretch respectively due to the geometry of the chamber. Thus, the non-uniformity of the pre-stretch is considered to be negligible. The distribution of the pre-stretch $\lambda_{0}$ obtained by ABAQUS is presented in Figure S4d. 
(a)

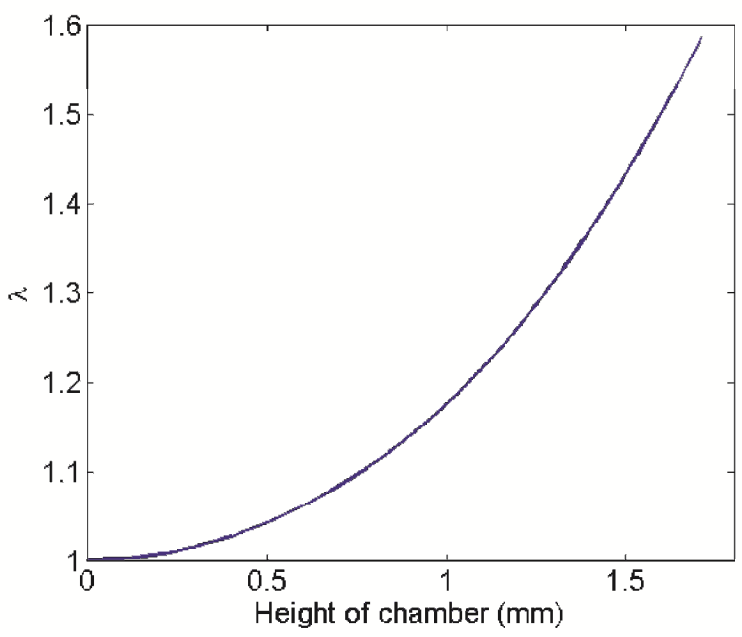

(b)

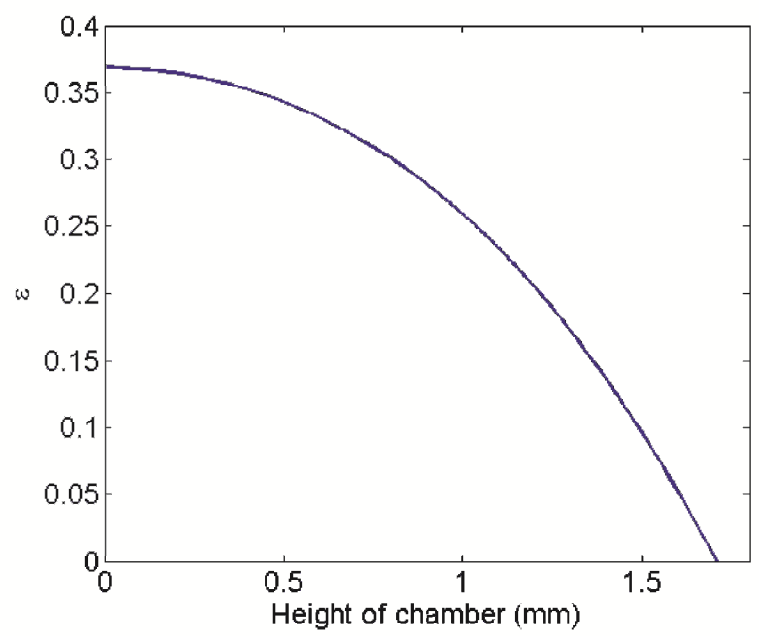

(c)

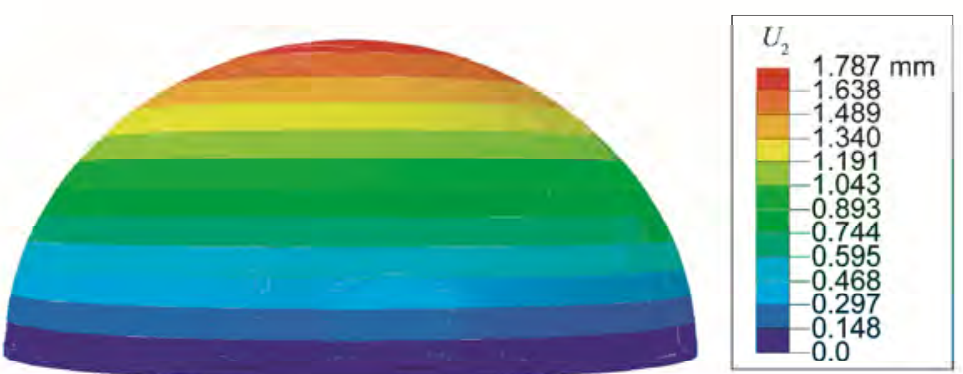

(d)

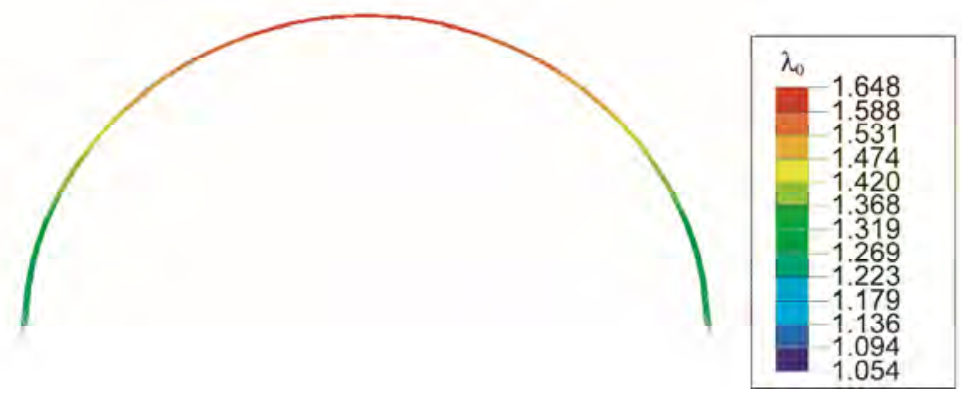




\section{Figure S4. Estimation of residual stress in Parylene film}

To estimate the residual stress and strain in the Parylene film, the radius of curvature of a free-standing Parylene/PDMS strip has been measured. A $150 \mathrm{~nm}$ thick Parylene film is deposited onto a $250 \mu \mathrm{m}$ thick free-standing PDMS substrate, and a $8 \mathrm{~mm}$ x $30 \mathrm{~mm}$ strip is cut from the bi-layer sample. The residual tensile stress in Parylene film gives rise to the curvature as shown below. The radius of curvature of short side is $\rho=19.0 \mathrm{~mm}$. Based on the Stoney formula ${ }^{[\mathrm{S1}, \mathrm{S} 2]}$, the residual tensile stress $\sigma_{f}$ and strain $\varepsilon_{f}$ in Parylene film are given by $\sigma_{f}=E_{s} h_{s}{ }^{2} / 6 h_{f} \rho(1-v)$ and $\varepsilon_{f}=(1+v) \sigma_{f} / E_{f}$. We obtain $\sigma_{f}=7.3 \mathrm{MPa}$ and $\varepsilon_{f}=0.63 \%$.

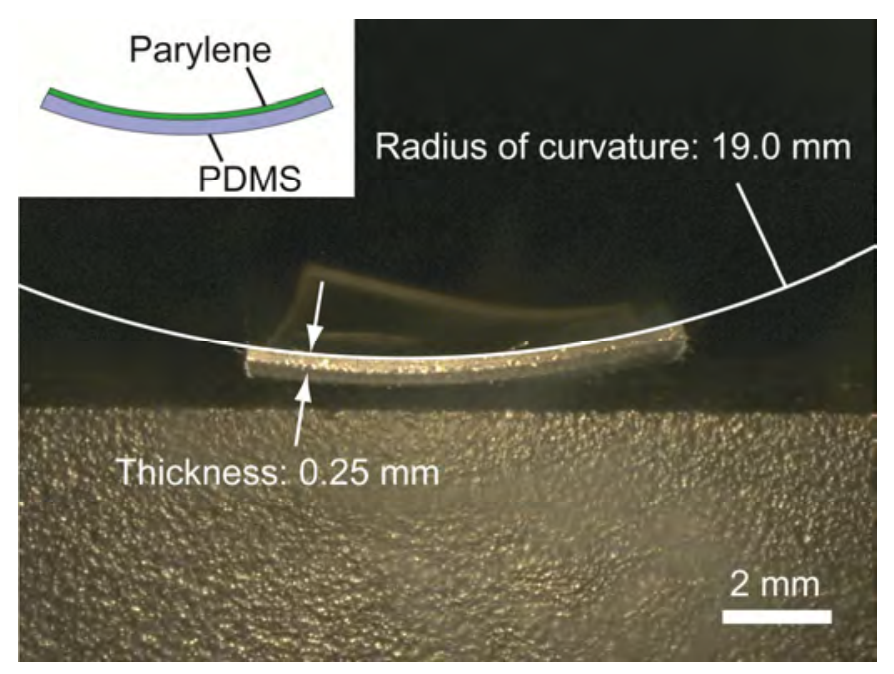

\section{Additional Reference}

[S1] G. G. Stoney, Proc. R. Soc. Lond. A, 1909, 82, 172

[S2] X. Feng, Y. Huang, A. J. Rosakis, Journal of Applied Mechanics, 2007, 74, 1276 